\title{
Correction of Accidental Patient Motion for Online Mr Thermometry
}

\author{
Baudouin Denis de Senneville ${ }^{1,2,3}$, Pascal Desbarats ${ }^{3}$, Rares Salomir ${ }^{4}$, \\ Bruno Quesson ${ }^{2}$, and Chrit T.W. Moonen ${ }^{2}$ \\ 1 IMF, ERT CNRS/Université Bordeaux 2 - 146, rue Léo Saignat, F-33076 Bordeaux \\ 2 Image Guided Therapy SA - 2, allée du doyen George Brus, F-33600 Pessac \\ 3 LaBRI, UMR 5800 CNRS/Université Bordeaux 1 - 351, cours de la Libération, \\ F-33405 Talence \\ 4 U386 INSERM, France
}

\begin{abstract}
Magnetic Resonance (MR) temperature mapping can be used to monitor temperature changes in minimally invasive thermal therapies during the procedure. The proton resonance frequency (PRF) shift technique gives an estimate of the relative temperature variation, comparing contrast between dynamically acquired images and reference data sets. However, organ displacements due to physiological activity (heart and respiration) may induce important artifacts on computed temperature maps if no correction is applied. This paper summarizes existing classical methods and presents a new approach for correction of accidental motion in such MR images in order to increase robustness of temperature estimation using the PRF shift. The correction method described in this paper consists of using image registration techniques to estimate motion on anatomical images.
\end{abstract}

\section{Introduction}

Local hyperthermia can be used for a wide variety of medical interventions such as tumor ablation [1], treatment of heart arrhythmias [2], local drug delivery with thermosensitive microcarriers 3] and control of gene therapy using heatsensitive promoters [4]. These interventions are usually monitored by CT scanner or ultrasound imaging (for the placement of the RF needle or the laser probe for example). However, MRI is the only technique that can provide on line quantitative thermometric information in addition to detailed anatomical information. It has been shown that tumor cell destruction occurs with a temperature increase included between $13^{\circ} \mathrm{C}$ and $23^{\circ} \mathrm{C}$ for a time treatment of several minutes [5]. The main principle of MRI is based on the detection of magnetic properties of the protons contained in the water molecules of the body 6]. The MR imaging system associates each volume unit of the target region of interest with a complex number $M e^{i \phi}$, where $M$ is the magnitude and $\phi$ the phase. The corresponding graylevel of the anatomical image is proportional to $M . \phi$ is then used to construct a phase image (cf Fig. 1). 
Under well-controlled conditions, a difference of phase between two subsequent images is proportional to a difference of temperature [9], as shown in Fig. 2 (the arrow in the second phase of the figure highlights a phase contrast due to a local heating of the sample using a MR-compatible focused ultrasound (FUS) device). However as temperature maps result from phase differences, this technique is prone to motion artifact [7]. In addition, local magnetic susceptibility is generally not fully uniform and the resulting phase variation $\Delta \varphi$ can be described by the following equation [8] :

$$
\Delta \varphi=\gamma \cdot \alpha \cdot B_{0} \cdot \Delta T \cdot T_{E}+\gamma \cdot B_{0} \cdot F T^{-1}\left[\left(\frac{1}{3}-\frac{K_{z}^{2}}{K^{2}}\right) \cdot F T(\Delta \chi)\right] \cdot T_{E}
$$

where $\Delta T$ is the temperature difference, $\gamma$ the gyromagnetic ratio $(\simeq 42.58$ $\mathrm{MHz} / \mathrm{T}), \alpha(=0.01 \mathrm{ppm} / K)$ the temperature coefficient, $T_{E}$ the echo time, $B_{0}$ the main magnetic field, $K=\left(K_{x}, K_{y}, K_{z}\right)$ the position vector in the reciprocal space, and $\Delta \chi$ the modification of the susceptibility field. The first term of the sum is due to the PRF shift with temperature whereas the second term rises when an object motion occurs.
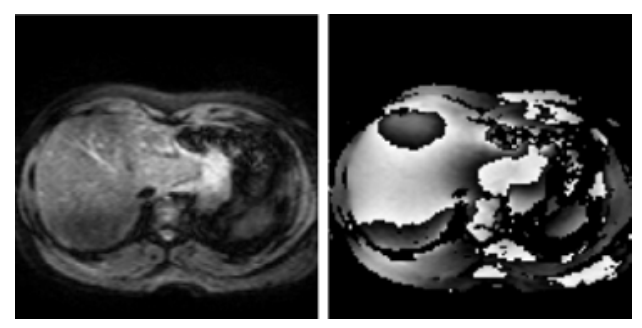

Fig. 1. Transverse magnitude and phase images of a human abdomen
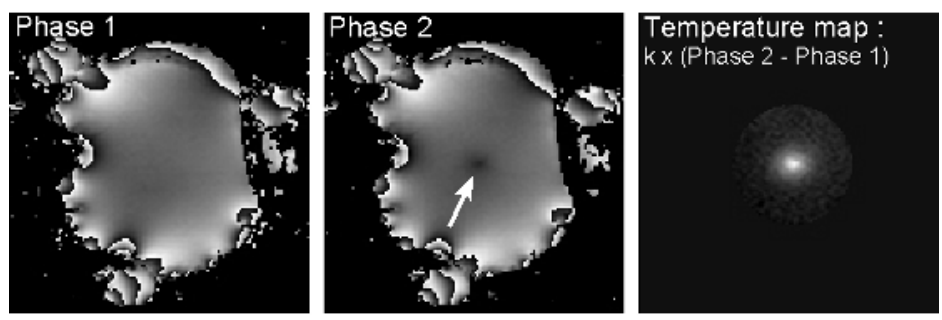

Fig. 2. Example of temperature map calculated on an ex-vivo muscle

Both PRF-shift with temperature and motion of the patient result in phase variation and it is not possible to separate these two contributions within a single phase image. Alternatively, it is possible to process magnitude images to extract and correct motion contribution to phase artifacts.

The case of uniform susceptibility object has already been studied [10]. However, modeling of inhomogeneous field of susceptibility in-vivo is difficult and 
this issue has to be addressed for clinical application. The approach described here allows to correct motion related errors in PRF based MR thermometry, avoiding explicit modeling of the susceptibility field.

For real time monitoring of temperature evolution, data treatment must be performed within the time duration between two successive acquisitions (i.e. in the range of $0.5 \mathrm{~s}$ to $1 \mathrm{~s}$ for a single $128 \times 128$ image for a standard thermometry sequence). Our test platform is an Athlon $2400 \mathrm{MHz}$ with 512Mo of RAM.

\section{Physical Approaches}

\section{$2.1 \quad$ Respiratory Gating}

Respiratory gating is a technique that allows reduction of artifacts due to respiration. The principle is to synchronize the acquisition of the images to a stable period of the respiratory cycle, usually at the end of expiration [11. The major drawback of this method is that the temporal resolution depends on the respiration frequency. Moreover, artifacts due to irregular and/or deep respiration movements may induce erroneous temperature mapping.

\subsection{Navigator Echoes}

Navigator echoes uses an approach that consists of acquiring one or multiple lines of Fourier space including the center and applying an inverse Fourier transformation in order to obtain the profile of the object. Navigator echoes can be used in two different ways :

Synchronization. It is possible to synchronize the acquisition of the images to a stable period of the respiratory cycle by using the profile of the object provided by the navigator echo technique. This approach is more efficient than the respiratory gating because the synchronization is done by using directly the MR signal on the studied region. Irregular and/or deep respiration movements thus are not limitations. On the other hand, as for the respiratory gating technique, the temporal resolution depends on the respiration frequency.

Motion estimation and correction. The profile of the object given by the navigator echoes can also be used to estimate translation of the object [12. This motion estimation is used to correct for temperature maps. If a motion occurs between $t_{n-1}$ and $t_{n}$, the relative temperature map after motion will be erroneous. A simple method consists of detecting motion vector to correct phase and temperature maps. Then the phase image acquired at $t_{n}\left(\varphi_{n}\right)$ is taken as new phase image reference, and the temperature mapping at $t_{i}\left(t_{i} \geq t_{n}\right)$ is computed with :

$$
\Delta T_{i}=\Delta T^{\prime}+\left(\varphi_{i}-\varphi_{n}\right) \cdot k
$$

where $\Delta T^{\prime}$ is is the $\mathrm{n}$-th temperature map after motion correction. 
However, the use of navigator echoes is restricted to rigid body motion and may not be very efficient for complex organs displacements such as in the abdomen (liver, kidney). Thus, accidental patient motion can't be corrected efficiency with this technique.

\subsection{Multi-baseline}

A collection of multiple baseline images can be use to generate temperature maps [13. Baseline selection can be made based on navigator echoes data [14].

\section{Computational Approaches}

Our approach consists of using image processing techniques to detect on line the displacements on the anatomic images in order to correct accidental patient motion. The objective is to relate the coordinate of each part of tissue in the image we want to register with the corresponding tissue in the reference image.

\subsection{Motion Estimation Techniques}

Methods based on a parameterized model of motion. The objective of this method is to determine a set of parameters for which a predetermined function of the parameters is minimized (or maximized). Optimum parameters can be calculated for example with the "Square Root Optimization" [15]).

"Block-matching" algorithm. This technique is used in the compression of videos in the MPEG format. The image to be registered is divided in blocks and the translation of each blocks in a research window is estimated [16]. It is supposed that the motion in the image is constant in a region.

Optical flow. This technique consists of extracting a displacement vector map from an image sequence assuming that intensity is conserved during displacement. Many methods can provide an estimation of the optical flow such as correlation methods (consists of establishing a correspondence between two images by localization of invariant markers [17]) and differential methods established by Horn and Schunck [18].

\subsection{Choice of Motion Estimation Method}

The most restrictive registration techniques are methods using parameterized models because the algorithm calculates a global transformation. The identification of the movement is done by the best possible adaptation of the parameters of the model (for example, there are six parameters to estimate in an affine model). The choice of the model requires to know the nature of the movement 
to obtain reliable results. The "Block-matching" technique is less restrictive because it corrects for local motion estimation. Therefore there is no limitation to the transformation that can be applied to the original image. The most permissive method is the calculation of the optical flow because the transformation is calculated on the vicinity of the pixels.

The choice of the registration method depends on which part of the body is observed [7. In this article, motion of the kidney was shown to be correctly described using a global affine transformation and a "Square Root Optimization" algorithm.

\subsection{Motion Correction}

The correction technique is nearly the same as for navigator echoes $(2.2)$ excepting that motion is directly estimated on anatomical image using image registration algorithms. The motion corrected temperature difference after the movement is added to temperature before movement. As an advantage, this technique is thus not limited to rigid body motion. Thus, this method is able to correct accidental patient motion and is complementary with periodic motion reduction techniques like respiratory gating.

\subsection{Experimental Validation}

Initial tests were performed on MR images acquired on a piece of an ex-vivo turkey muscle heated using a laser device (Dornier). 14 slices have been acquired each $15.3 \mathrm{~s}$ by the MRI. The spatial resolution of those images was $128 \times 128$ pixels and the pixel size was $1 \times 1 \mathrm{~mm}$. During the heating process, a mechanical translation and rotation was applied.

A second experiment was performed on a healthy volunteer under free breathing. 3 slices have been acquired using respiratory gating (each $5 \mathrm{~s}$ approximatively). The spatial resolution of those images was $128 \times 128$ pixels and the pixel size was $1.4 \times 1.4 \mathrm{~mm}$. In the middle of the acquisition, the volunteer was asked to make one body displacement and to stay in final position. The experiment consisted of measuring the stability of the thermometry baseline in the kidney of a healthy volunteer using our approach.

\section{Results and Discussion}

\subsection{Motion Correction on an Ex-vivo Muscle Heated with a Laser Device}

Figure 3 shows the anatomical image of the muscle (3) A) and the temperature mapping measured during the heating process when no movement occurs 3 B), when a movement occurs and no correction has been achieved (3. C), and with the same movement when our correction is applied (3. D). Motion was estimated with the "Square Root Optimization" algorithm using a global 3D 
rigid transformation and required 2.8s of computation time for the entire volume on our test platform. Figure 4 shows the temporal evolution of the temperature measured on a pixel situated on the heating zone of the muscle. A movement occurs after 250 s and the heating process has been stopped after 350 s.
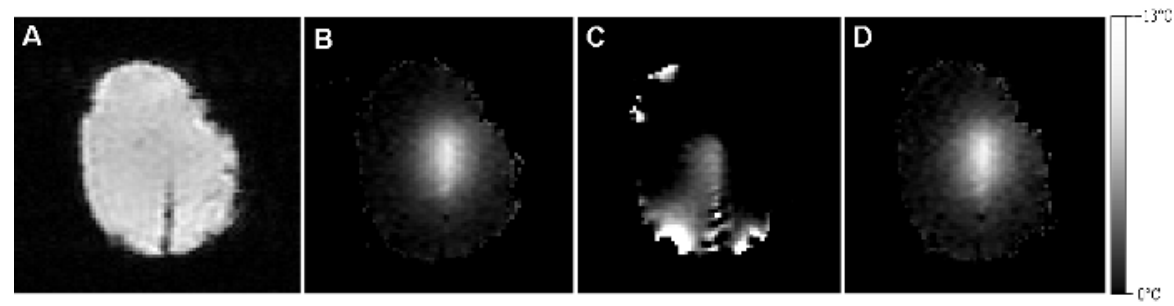

Fig. 3. Motion correction on an ex-vivo muscle

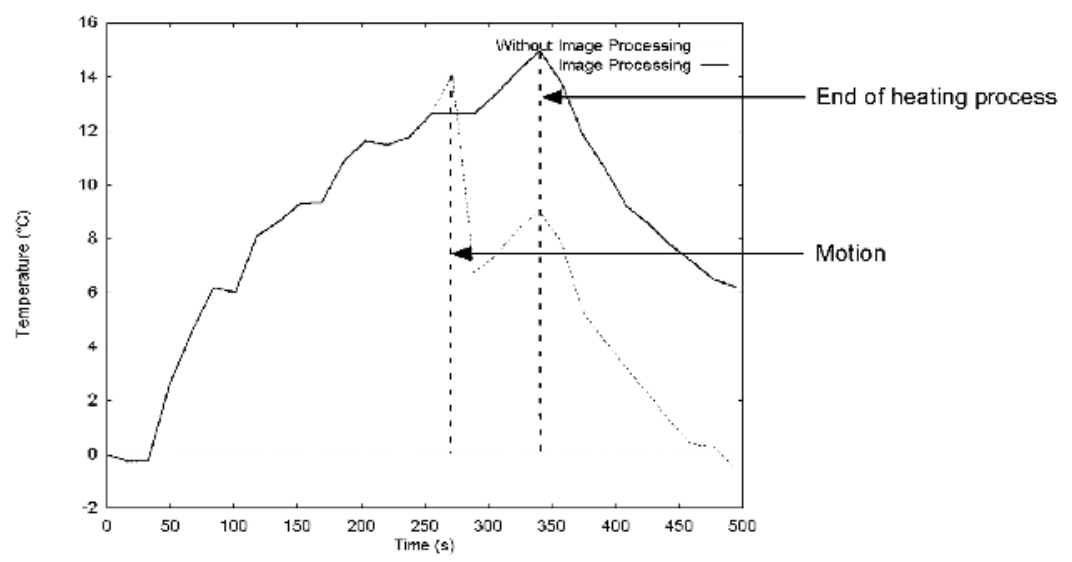

Fig. 4. Temporal evolution of the temperature on a pixel located in the heated zone

\subsection{Motion Correction on a Healthy Volunteer}

Figure 5 shows anatomical images obtained when no movement occurs 5. A) and when a movement occurs (5. B). Figure 5C shows the temperature mapping measured when a movement occurs and no correction has been achieved. Motion is estimated with the "Square Root Optimization" algorithm using a global 2D affine transformation and required $1.9 \mathrm{~s}$ of computation time for the 3 slices on our test platform. Figure 6 shows the temporal evolution of the temperature measured on a pixel situated on the kidney.

Experimental results demonstrated that this motion correction technique allowed a precise recovery of the temperature map. 

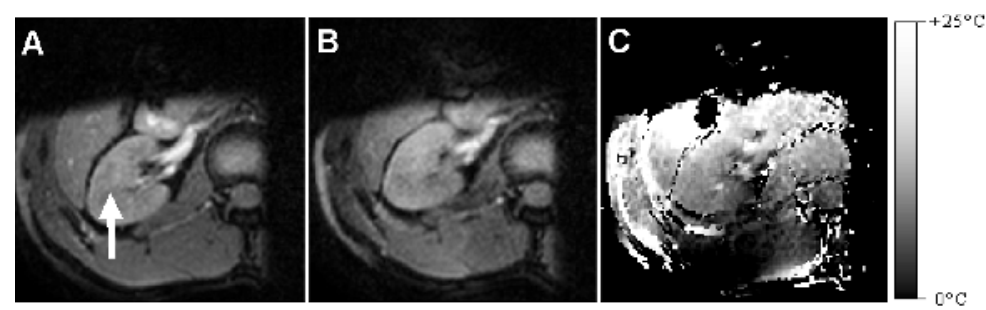

Fig. 5. Motion corrupted images in a healthy volunteer

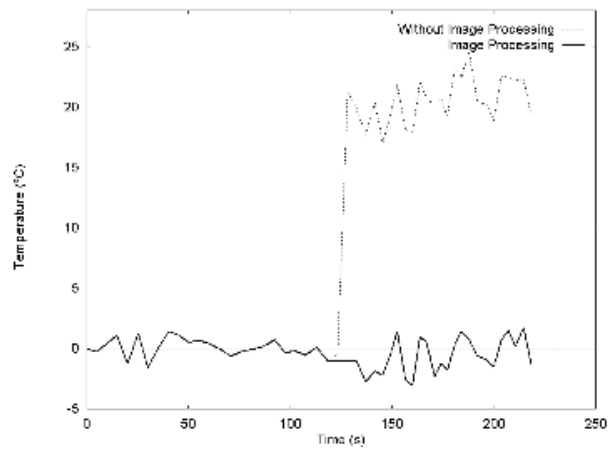

Fig. 6. Temporal evolution of the temperature on a pixel reported on Figure 5 A (white arrow)

\subsection{Discussion}

The implemented algorithm for image registration can be parameterized in order to perform on line temperature correction under these experimental conditions. Our approach allows to correct complex motion. This method must be combined to other techniques like respiratory gating to improve the stability of the thermometry.

Although this method can be used to correct for motion corrupted images during on line MR temperature mapping, several limitations remain. First, using this method information about temperature changes between the instant $t_{n-1}$ and $t_{n}$ is lost. Moreover, as mention in [12], noise in the temperature map increases with a number of motion artifacted images. In addition, uncertainty induced by the correction technique is reflected on all the images obtained after displacements. As a consequence, this technique can be applied when periodic motion has already been corrected with synchronization techniques. It has been demonstrated here that accidental displacement can be corrected in such cases. However, in the case of rapid MR acquisition without synchronization, added noise on phase images may dramatically hamper the quality of MR thermometry. This motion correction technique for accidental patient movements has a straightforward medical application in MR guided thermotherapies and further experiments are in progress including clinical cases. 


\section{References}

1. Steiner P., Botnar R., Dubno B. et al. Radio-frequency induced thermoablation: monitoring with T1-weighted and proton frequency shift MR imaging in an interventional 0.5-T environment. Radiology 1998;206:803-810.

2. Levy S. Biophysical basis and cardiac lesions caused by different techniques of cardiac arrhythmia ablation. Arch. Mal. Coeur Vaiss. 1995;88:1465-1469.

3. Kim S. Liposomes as carriers of cancer chemotherapy: current status and future prospects. Drugs 1993;46:618-638.

4. Madio D.P., van Gelderen P., DesPres D., et al. On the feasibility of MRI-guided focused ultrasound for local induction of gene expression. J. Magn. Res. Imaging 1998;8:101-104.

5. Sapareto S. A., Dewey W. CL, Thermal dose determination in cancer therapy. Int. J. Radiation Oncology Biol. Phys. 10, 787-800. 1984.

6. Mansfield P. et Grannell P.K. NMR "diffraction" in solids?. J. Phys. C:Solid state phys 1973;6,L422-L426.

7. Denis de Senneville B., Desbarats P., Quesson B., Moonen C. T. W., RealTime Artefact Corrections For Quantitative MR Temperature Mapping. Journal of WSCG, Vol.11, No.1.,ISSN 1213-6972. WSCG 2003, February 3-7,2003, Plzen, Czech Republic.

8. Salomir R., Denis de Senneville B., Moonen C. T. W., A fast calculation method for magnetic field inhomogeneity due to an arbitrary distribution of bulk susceptibility. Wiley InterScience. 7 July 2003.

9. Quesson B., de Zwart J. A., Moonen C. T. W., Magnetic Resonance Temperature Imaging for Guidance of Thermotherapy. Journal of Magnetic Resonance Imaging, 2000;12:523-533.

10. Vogel M. W., Suprijanto, Vos F.M., Vrooman H.A., Vossepoel A.M., Pattynama P.M.T., Towards motion-robust magnetic resonance thermometry. Miccai 2001.

11. Moricawa S, Inubushi T, Kurumi Y, Naka S, Seshan V, Tsukamoto T. Feasibility of simple respiratory triggering in MR-guided interventional procedures for liver tumors under general anesthesia.

12. de Zwart J. A., Vimeux F., Palussiére J., Salomir R., Quesson B., Delalande C., and Moonen C. T. W., On-Line Correction and Visualiszation of Motion During MRI-Controlled Hyperthermia. Magnetic Resonance in Medicine. 2001; 45:128-137.

13. Suprijanto, Vogel M.W., Vos F.M., Vrooman H.A., and Vossepoel A.M., Displacement Correction Scheme for MR-Guided Interstitial Laser Therapy, Medical Image Computing and Computer-Assisted Intervention. MICCAI 2003.

14. Vigen Karl K., Daniel Bruce L., Pauly John M., Butts Kim, Triggered, navigated, multi-baseline method for proton resonance frequency temperature mapping with respiratory motion. Magn Reson Med 50:1003-1010, 2003.

15. Friston K.J., Ashburner J, Frith C.D., Poline J-B., Heather J.D. and Frackowiak R.S.J. Spatial registration and normalisation of images. Human Brain Mapping. 2:165-189. 1995.

16. Chen M.J. Chen L.G., Chiueh T.D. \& Lee Y.P. A new block-matching criterion for motion estimation and its implementation. IEEE Transactions on Circuits and Sytems for Video Technology. 5:231-236. 1995.

17. Singh A., "An estimation-theoric framework for image-flow computation", Proc. 3rd Itern. Conf. Comput. Vis., Osaka, pp. 168-177, 1990.

18. Schunck B.G. Horn K.P. - Determining optical flow. Artificial intelligence, 17:pp. 185-203,1981. 